Pacific Journal of Mathematic 


\title{
ON THE INNER APERTURE AND INTERSECTIONS OF CONVEX SETS
}

\author{
D. G. LARMAN
}

If $C_{1}, \cdots, C_{n}$ are $n$ convex surfaces or sets in $d$-dimensional Euclidean space $E^{d}$, then it is of some interest to study the invariance properties of $\bigcap_{i=1}^{n}\left(C_{i}+\boldsymbol{a}_{i}\right)$ for all choices of vectors $\boldsymbol{a}_{i}$ in $E^{d}$. Such considerations occur naturally in identifying an object irrespective of the direction in which it approaches the observer.

For example, Melzak [2] and Lewis [1] have investigated the conditions under which the intersection $\bigcap_{i=1}^{d}\left(C_{i}+\boldsymbol{a}_{i}\right)$ of certain convex surfaces always is a single point. These surfaces arise from the work of Ratcliff and Hartline [3] concerning varying light intensities upon different visual elements of the eye.

In this article we study such intersections and in Theorem 1, we show that the result of Melzak [1] has an associated Helly number in $E^{2}$ but not in $E^{3}$. In Theorem 2 we give a necessary and sufficient condition for $\bigcap_{i=1}^{n} C_{i}+\boldsymbol{a}_{i}$ to be nonempty, whenever $C_{1}, \cdots, C_{n}$ are convex sets, in terms of the outward normals. This condition is not easy to apply in that it involves the outward normals to intersections of $d$-membered subsets. So in Theorem 3 we give a sufficient condition in terms of inner and outer apertures which is widely applicable. Finally, in Theorem 4, we give a characterization of the sets which can arise as inner apertures. I am indebted to Z. A. Melzak for suggesting these problems to me.

To define the inner and outer aperture, let $D$ be a convex subset of $E^{d}$. If $l \equiv l(u, v)$,

$$
l=\{\boldsymbol{u}+\lambda \boldsymbol{v}, \lambda \geqq 0\}
$$

is a typical ray in $E^{d}, \boldsymbol{u}, \boldsymbol{v} \in E^{d}, \boldsymbol{v} \neq \boldsymbol{o}$, define

$$
\theta(\lambda, D)=\text { dist. }\left\{\boldsymbol{u}+\lambda \boldsymbol{v}, E^{d} \backslash D\right\}
$$

and

$$
\theta(D)=\sup _{\lambda \geq 0} \theta(\lambda)
$$

where

$$
\text { dist. }\{A, B\}=\inf _{\substack{a \in A \\ b \in B}}\|\boldsymbol{a}-\boldsymbol{b}\|
$$

when $A, B$ are nonempty subsets of $E^{d}$. The inner aperture $\mathscr{J}(D)$ of $D$ is the union of those rays $l(u, v)-\boldsymbol{u}$ emanating from the origin 
o such that $\theta(l(u, v), D)=+\infty$. So, if $D$ contains $\boldsymbol{o}, \mathscr{J}(D)$ is the union of those rays $l \equiv l(\boldsymbol{o}, \boldsymbol{u})$ in $D$ such that $\lambda \boldsymbol{u}$ can be made an arbitrarily large distance from the boundary of $D$ for $\lambda$ sufficiently large. The outer cone $O(D)$ of $D$ is what is usually known as the characteristic cone namely the set of all rays $l(u, v)-u$ emanating from $\boldsymbol{o}$ with $l(\boldsymbol{u}, \boldsymbol{v})$ contained in $D$. Both $O(D)$ and $\mathscr{I}(D)$ are convex cones and $O(D)$ is closed whenever $D$ is closed. In general, of course, $O(D)$ can be any convex cone in $E^{d}$ but this is not the case for $\mathscr{J}(D)$. It will follow from Theorem 4 that $\mathscr{J}(D)$ is a $G_{0}$-convex cone with the property that whenever a ray $l \in \operatorname{cl} .\{\mathscr{J}(D)\} \mid \mathscr{I}(D)$ then the smallest exposed face $F(l)$ of cl. $\{\mathscr{J}(D)\}$ containing $l$ also is contained in $\{$ cl. $\mathscr{I}(D)\} \mid \mathscr{I}(D)$.

Theorem 1. Let $C_{1}^{*}, \cdots, C_{n}^{*}$ be $n$ convex sets in $E^{d}$ whose $d$ dimensional interiors are nonempty and do not contain a line. Let $C_{1}$, $\cdots, C_{n}$ be the convex surfaces bounding $C_{1}^{*}, \cdots, C_{n}^{*}$ respectively. Then $\bigcap_{j=1}^{n}\left(C_{j}+\boldsymbol{a}_{j}\right)$ is at most a single point for all choices $\boldsymbol{a}_{1}, \cdots, \boldsymbol{a}_{n}$ of points in $E^{d}$ if and only if there does not exist $n$ parallel lines of support $l_{1}, \cdots, l_{n}$ to $C_{1}^{*}, \cdots, C_{n}^{*}$ respectively. In $E^{2}$ this is true if and only if some four membered subset $C_{j_{1}}^{*}, \cdots, C_{j_{4}}^{*}$ do not have parallel lines of support. However, in $E^{3}$ and for every $n \geqq 3$ there exist convex sets $C_{1}^{*}, \cdots, C_{n}^{*}$, whose relative interiors do not contain a line, such that every $n-1$ membered subset have parallel lines of support but this is not so for $C_{1}^{*}, \cdots, C_{n}^{*}$.

Lemma 1. Let $A_{1}, \cdots, A_{n}$ be spherically convex subsets (possibly open, half-open or closed semicircles) of the unit circle $S^{1}$ such that

$$
\bigcap_{\nu=1}^{4}\left(A_{i_{\nu}} \cup-A_{i_{\nu}}\right) \neq \varnothing, 1 \leqq i_{\nu} \leqq n, \nu=1, \cdots, 4 .
$$

Then

$$
\bigcap_{i=1}^{n}\left(A_{i} \cup-A_{i}\right) \neq \varnothing
$$

Proof. We parametrise $S^{1}$ in terms of the angle $\theta$ made with some fixed line through the origin and consider the semicircular interval $[0, \pi]$. The intersection $A_{i} \cup-A_{i}$ with $[0, \pi]$ is either

(i) an interval $\left\langle c_{i}, d_{i}\right\rangle$ not containing either 0 or $\pi$, or (ii) $[0, \pi]$,

or (iii) two intervals $\left[0, a_{i}>,<b_{i}, \pi\right]$, the first containing 0 and the second containing $\pi$.

The classification yields a corresponding subdivision $I_{1}, I_{2}, I_{3}$ of $\{1, \cdots, n\}$. Let 


$$
\begin{aligned}
& {\left[0, a_{i_{1}}\right\rangle=\bigcap_{i \in I_{3}}\left[0, a_{i}\right\rangle} \\
& \left\langle b_{i_{2}}, \pi\right]=\bigcap_{i \in I_{3}}\left\langle b_{i}, \pi\right] .
\end{aligned}
$$

If $\left\langle c_{i}, d_{i}\right\rangle$ and $\left\langle c_{j}, d_{j}\right\rangle, i, j \in I_{1}$ both meet $\left[0, a_{i_{1}}\right\rangle$ and

$$
\left\langle c_{i}, d_{i}\right\rangle \cap\left\langle c_{j}, d_{j}\right\rangle \cap\left[0, a_{i_{1}}\right\rangle=\varnothing
$$

then at least one of these intervals is contained in $\left[0, a_{i_{1}}\right\rangle$. But then

$$
\left(A_{i} \cup-A_{i}\right) \cap\left(A_{j} \cup-A_{j}\right) \cap\left(A_{i_{1}} \cup-A_{i_{1}}\right) \cap\left(A_{i_{2}} \cup-A_{i_{2}}\right)
$$

is contained in $\left[0, a_{i_{1}}\right\rangle \cup-\left[0, a_{i_{1}}\right\rangle$ and consequently, by (1), is empty, which is contradiction. So, if

$$
I_{1}^{1}=\left\{i \in I_{1}:\left\langle c_{\imath}, d_{i}\right\rangle \cap\left[0, a_{i_{1}}\right\rangle \neq \varnothing\right\}
$$

we have, from Helly's theorem, that

$$
\left[0, a_{i_{1}}\right\rangle \cap \bigcap_{i \in I_{1}^{1}}\left\langle c_{i}, d_{i}\right\rangle \neq \varnothing
$$

Similarly, if

$$
\begin{gathered}
I_{1}^{2}=\left\{i \in I_{1}:\left\langle c_{i}, d_{i}\right\rangle \cap\left\langle b_{i_{2}}, \pi\right] \neq \varnothing\right\} \\
\left\langle b_{i_{2}}, \pi\right] \cap \bigcap_{i \in I_{1}^{2}}\left\langle c_{i}, d_{i}\right\rangle \neq \varnothing .
\end{gathered}
$$

If there exists $i_{3} \in I_{1} \backslash I_{1}^{1}$ and $i_{4} \in I_{1} \backslash I_{1}^{2}$ then

$$
\bigcap_{\nu=1}^{4} A_{i_{\nu}} U-A_{i_{\nu}}=\varnothing,
$$

so either $I_{1}^{1}=I_{1}$ or $I_{1}^{2}=I_{1}$ and, using (2) and (3),

$$
\bigcap_{i=1}^{n} A_{i} \cup-A_{i} \neq \varnothing \text {. }
$$

REMARK. This is the best possible result for if $A_{1}=[0, \pi / 2], A_{2}=$ $[\pi / 4,3 \pi / 4], A_{3}=[\pi / 2, \pi], A_{4}=[3 \pi 4,5 \pi / 4]$ then

$$
\bigcap_{u=1}^{3} A_{i_{\nu}} \cup-A_{i_{\nu}} \neq \varnothing, 1 \leqq i_{1}<i_{2}<i_{3} \leqq 4
$$

but

$$
\bigcap_{i=1}^{4} A_{i} \cup-A_{i}=\varnothing \text {. }
$$

LEMMA 2. There exist $n$ closed spherically convex two dimensional subsets $D_{1}, \cdots, D_{n}$ on $S^{2}$, none of which contain antipodal points, such that for every $n-1$ membered subset $D_{i_{1}}, \cdots, D_{i_{n-1}}$ there exists 
a great circle of $S^{2}$ which meets each $D_{i_{\nu}}$, but there does not exist a great circle meeting each of $D_{1}, \cdots, D_{n}$.

Proof. In [4], Santalo constructs, for each $n \geqq 3$, a family of $n$ compact convex two dimensional sets $F_{1}, \cdots, F_{n}$ in $E^{2}$ so that each $n-1$ members of the family admit a common transversal but the entire family does not have a common transversal. We mention that such an example is the family of $n$ circular discs whose centers have polar coordinates $\rho=1$ and $\theta=2 k \pi / n, k=1, \cdots, n$ and whose radii are all equal to $\cos ^{2} \pi / n$ or $\cos ^{2} \pi / n+\cos ^{2} \pi / 2 n-1$ according as whether $n$ is even or odd.

Now, if we place the configuration $F_{1}, \cdots, F_{n}$ into a plane tangent to $S^{2}$, let $D_{1}, \cdots, D_{n}$ be the corresponding closed spherically convex subsets of $S^{2}$ obtained by the projection of $F_{1}, \cdots, F_{n}$ into $S^{2}$ from the origin. Clearly $D_{1}, \cdots, D_{n}$ satisfy the requirements of the lemma.

Proof of Theorem 1. The proof of the first part is essentially due to Melzak [1] but as he makes the restriction that $d=n$ we repeat the details.

If there exist $n$ parallel lines of support $l_{1}, \cdots, l_{n}$ to $C_{1}^{*}, \cdots, C_{n}^{*}$ respectively then by translating the line $l_{j}$ into the relative interior of $C_{j}$ if necessary, $j=1, \cdots, n$ we obtain $n$ nondegenerate similarly orientated chords $\left[\boldsymbol{p}_{j}, \boldsymbol{q}_{j}\right]$ of $C_{j}^{*}$ parallel to $l_{j}$ such that

$$
\left\|\boldsymbol{p}_{1}-\boldsymbol{q}_{1}\right\|=\cdots=\left\|\boldsymbol{p}_{n}=\boldsymbol{q}_{n}\right\| \text {. }
$$

Hence, if $\boldsymbol{a}_{j}=\boldsymbol{p}_{i}-\boldsymbol{p}_{j}, j=1, \cdots, n$

$$
\bigcap_{j=1}^{n} C_{j}^{*}+\boldsymbol{a}_{j} \supset\left\{\boldsymbol{p}_{1}, \boldsymbol{q}_{1}\right\}
$$

and so contains at least two points.

On the other hand, if there exist vectors $\boldsymbol{a}_{j}, j=1, \cdots, n$ such that $\bigcap_{j=1}^{n} C_{j}^{*}+\boldsymbol{a}_{j}$ contains at least two points say $\boldsymbol{p}, \boldsymbol{q}$ then, by considering two dimensional sections of $C_{j}, C_{j}$ has a line of support $l_{j}$ parallel to $[\boldsymbol{p}, \boldsymbol{q}]$ and hence $l_{1}, \cdots, l_{n}$ are parallel lines of support to $C_{1}, \cdots, C_{n}$ respectively which completes the proof of the first part.

In $E^{2}$ we may select a set $A_{i}$ of unit tangent vectors $\boldsymbol{u}$ to $C_{i}^{*}$ by ensuring that the outward normal lies on the left hand side of $\boldsymbol{u}$ when viewed from the point of contact on $C_{i}$ in a clockwise direction. Then $A_{i}$ is a spherically convex subset of $S^{1}$ which is either $S^{1}$ or is contained in semicircle according to whether or not $C_{i}$ is bounded. Now $C_{1}^{*}, \cdots, C_{n}^{*}$ do not have parallel lines of support if and only if

$$
\bigcap_{i=1}^{n}\left(A_{i} \cup-A_{i}\right)=\varnothing \text {. }
$$


This, by Lemma 1 , is true if and only if there exists some four membered subset of $C_{1}^{*}, \cdots, C_{n}^{*}$ which do not possess parallel lines of support which completes the proof of the second part of the theorem.

In $E^{3}$ and for each $n \geqq 2$ consider the $n$ closed spherically convex subsets $D_{1}, \cdots, D_{n}$ of $S^{2}$ afforded by Lemma 2 . If $\langle$,$\rangle denotes scalar$ product consider the set of closed half-spaces $\mathscr{H}_{i}$ such that $H^{-} \in \mathscr{H}_{i}$ if

$$
H^{-}=\{\boldsymbol{x}:\langle\boldsymbol{x}, \boldsymbol{u}\rangle \leqq 1\} \text { for some } \boldsymbol{u} \in D_{i} .
$$

Let

$$
C_{i}^{*}=\bigcap_{\mathscr{C}_{i}} H^{-}, \quad i=1, \cdots, n .
$$

Then $D_{i}$ is the set of outward normals to $C_{i}^{*}$ and so as $D_{i}$ is two dimensional, $C_{i}^{*}$ does not contain a line, $i=1, \cdots, n$. Also for every $n-1$ membered subset $C_{i_{i}}^{*}, \cdots, C_{i_{n-1}}^{*}$ of $C_{1}, \cdots, C_{n}$ the corresponding set of outward normals $D_{i_{1}}, \cdots, D_{i_{n-1}}$ all meet some great sphere $S \equiv$ $S\left(i_{1}, \cdots, i_{n-1}\right)$. Consequently, if $l$ is a line perpendicular to aff. $S$, $C_{i_{1}}, \cdots, C_{i_{n-1}}$ each possess lines of support parallel to $l$.

On the other hand, if $C_{1}, \cdots, C_{n}$ possess parallel lines of support then there would exist a great sphers $S^{1}$ of $S^{2}$ which meets each of $D_{1}, \cdots, D_{n}$ which, by Lemma 2 , is not so. Hence $C_{1}, \cdots, C_{n}$ do not possess parallel lines of support, which completes the proof of Theorem 1.

We observe the following lemma which is easily established by separating two disjoint convex sets by a hyperplane.

Lemma 3. Two convex sets $C_{1}, C_{2}$ in $E^{d}$ cannot be separated by translation if and only if $N\left(C_{1}\right) \cap\left(-N\left(C_{2}\right)\right)=$ o, where $N\left(C_{i}\right)$ is the convex cone of outward normals to $C_{i}, i=1,2$.

Using Helly's theorem we readily verify the following lemma.

Lemma 4. If $C_{1}, \cdots, C_{n}$ are convex sets in $E^{d}$, then $\bigcap_{i=1}^{n}\left(C_{i}+\right.$ $\left.\boldsymbol{a}_{i}\right) \neq \varnothing$ for all points $\boldsymbol{a}_{1}, \cdots, \boldsymbol{a}_{n}$ in $E^{d}$ if and only if $\bigcap_{v=1}^{d+1}\left(C_{i_{\nu}}+\boldsymbol{a}_{i_{\nu}}\right) \neq$ $\varnothing$ for all points $\boldsymbol{a}_{1}, \cdots, \boldsymbol{a}_{n}$ in $E^{d}$ and for every $d+1$ membered subset $\left\{C_{\imath_{2}}\right\}_{u=1}^{d+1}$ of $\left\{C_{i}\right\}_{i=1}^{n}$.

Using Lemmas 3 and 4 we obtain

Theorem 2. If $C_{1}, \cdots, C_{n}$ are convex sets in $E^{d}$ then $\bigcap_{i=1}^{n}\left(C_{i}+\right.$ $\left.\boldsymbol{a}_{i}\right) \neq \varnothing$ for all points $\boldsymbol{a}_{1}, \cdots \boldsymbol{a}_{n}$ in $E^{d}$ if and only if

$$
\left\{-N\left(C_{i_{1}}\right)\right\} \cap N\left(\bigcup_{\nu=2}^{d+1} C_{i_{\nu}}\right)=\varnothing
$$


for all $d+1$ membered subcollections $\left\{C_{i_{\nu}}\right\}_{v=1}^{d+1}$ of $\left\{C_{i}\right\}_{i=1}^{n}$.

However, this condition is not completely satisfactory in that $N\left(\bigcup_{v=2}^{d+1} C_{i_{\nu}}\right)$ is a function of $\bigcup_{v=2}^{d+1} C_{i_{\nu}}$ rather than a combination of functions of each $C_{i_{\nu}}$. We shall resolve this problem to a certain extent in Theorem 3 by giving a widely applicable sufficient condition.

Theorem 3. Let $C_{1}, \cdots, C_{n}$ be $n$ convex sets in $E^{d}$. Then

$$
\bigcap_{i=1}^{n}\left(C_{i}+\boldsymbol{a}_{i}\right) \neq \varnothing
$$

for all choices of $\boldsymbol{a}_{1}, \cdots, \boldsymbol{a}_{n}$ if there exists $j$ such that

$$
O\left(\text { cl. } C_{j}\right) \cap \bigcap_{i=1}^{d+1} \mathscr{J}\left(C_{i_{\nu}}\right) \neq \varnothing
$$

for all $d+1$ membered subcollections $\left\{C_{i_{\nu}}\right\}_{0=1}^{d+1}$ of $\left\{C_{i}\right\}_{\substack{i=1 \\ i \neq j}}^{n}$ Further, if at least of cl. $C_{1}, \cdots, \mathrm{cl} . C_{n}$ does not contain a line, each is unbounded and $C_{1}, \cdots, C_{n}$ cannot be separated by translation, i.e., (4) holds for all $\boldsymbol{a}_{1}, \cdots, \boldsymbol{a}_{n}$ then

$$
\bigcap_{j=1}^{n} O\left(\mathrm{cl} . C_{j}\right) \neq \varnothing
$$

Proof. Let $l$ be a ray of $O\left(\right.$ cl. $\left.C_{j}\right) \cap \bigcap_{i=1}^{n} \mathscr{J}\left(C_{i}\right)$ which, by Helly's theorem, is nonempty. We may suppose, without loss of generality, that $\boldsymbol{o} \in C_{1} \cap \cdots \cap C_{n}$. Then, if $\boldsymbol{a}_{1}, \cdots, \boldsymbol{a}_{n}$ are points of $E^{d}$,

$$
l+\boldsymbol{a}_{i} \subset C_{i}+\boldsymbol{a}_{i}, \quad i=1, \cdots, n .
$$

If $l=\{\lambda u, \lambda \geqq 0\}$, then, as $l \subset \mathscr{F}\left(C_{i}\right), i \neq j$, there exists $\lambda_{i}$ such that $\lambda \boldsymbol{u}+\boldsymbol{a}_{j}$ is in $C_{i}, \lambda \geqq \lambda_{i}$.

So, if $\lambda^{*}=\max _{1 \leqq i \leqq n} . \lambda_{i}$,

$$
\lambda^{*} u+a_{j} \in \bigcap_{i=1}^{n} C_{i} \text { as required . }
$$

To prove the second part, let $C_{i}^{*}$ denote the closure of $C_{i}, i=1$, $\cdots, n$. We may assume that $C_{1}$ and $C_{1}^{*}$ do not contain a line and that for some $n, \bigcap_{i=1}^{n-1} C_{i}^{*}$ is unbounded, which is certainly true for $n=2$. As $\bigcap_{i=1}^{n-1} C_{i}^{*}$ is convex closed and unbounded it follows that $O\left(\bigcap_{i=1}^{n-1} C_{i}^{*}\right)$ is nonempty. Further, as $\bigcap_{i=1}^{n-1} C_{i}^{*}$ is contained in $C_{1}^{*}$, $\bigcap_{i=1}^{n-1} C_{i}^{*}$ and $O\left(\bigcap_{i=1}^{n-1} C_{i}^{*}\right)$ do not contain a line. Let $l$ be a ray of $O\left(\bigcap_{i=1}^{n-1} C_{i}^{*}\right)$, say $l=\{\lambda u, \lambda \geqq 0\}$. If $O\left(\bigcap_{i=1}^{n} C_{i}^{*}\right)$ is empty then, in particular, $\bigcap_{i=1}^{n} C_{i}^{*}$ must be a compact convex set.

If $\lambda \geqq 0$,

$$
\lambda \boldsymbol{u}+\bigcap_{i=1}^{m-1} C_{i} \subset \bigcap_{i=1}^{m-1} C_{i}
$$


and consequently,

$$
\left(\lambda \boldsymbol{u}+\bigcap_{i=1}^{m-1} C_{i}\right) \cap C_{m}=\left(\lambda \boldsymbol{u}+\bigcap_{i=1}^{m-1} C_{i}\right) \cap\left(\bigcap_{i=1}^{m} C_{i}\right) .
$$

If no matter how large $\lambda$ is taken, $\left(\lambda \boldsymbol{u}+\bigcap_{i=1}^{m-1} C_{i}\right) \cap C_{m}$ contains a point $z(\lambda)$ say then, by (5), $z(\lambda)$ is confined to a compact set $\bigcap_{i=1}^{m} C_{i}$ and $z(\lambda)-\lambda \boldsymbol{u} \in \bigcap_{i=1}^{m-1} C_{i}, \lambda \geqq 0$. It follows that $-l$ is a ray of $O\left(\bigcap_{i=1}^{m-1} C_{i}^{*}\right)$ which is a contradiction to $C_{1}^{*}$ not containing a line. So $\bigcap_{i=1}^{m} C_{i}^{*}$ is an unbounded closed convex set and hence $O\left(\cap_{i=1}^{m} C_{i}^{*}\right)$ is nonempty. So repeating this process for $m=1,2, \cdots, n$ we conclude that $O\left(\cap_{i=1}^{n} C_{i}^{*}\right)$ is nonempty as required.

Definition. We say that a collection $\mathscr{H}$ of closed half-spaces in $E^{d}$ is closed if whenever $\left\{H_{i}^{-}\right\}_{i=1}^{\infty}$ is a sequence of closed half-spaces in $\mathscr{H}$, where

$$
H_{i}^{-}=\left\{\boldsymbol{x}:\left\langle\boldsymbol{x}, \boldsymbol{u}_{i}\right\rangle \leqq \alpha_{i}\right\}, \boldsymbol{u}_{i} \text { a unit vector },
$$

and $\boldsymbol{u}_{i} \rightarrow \boldsymbol{u}, \alpha_{i} \rightarrow \alpha$ as $i \rightarrow \infty$ then the closed half-space

$$
H^{-}=\{\boldsymbol{x}:\langle\boldsymbol{x}, \boldsymbol{u}\rangle \leqq \alpha\}
$$

is in $\mathscr{H}$. We say that a collection $\mathscr{H}$ of closed half-spaces is $F_{\sigma}$ if it is the countable union of closed collections.

If $\mathscr{H}$ is a closed collection of closed half-spaces notice that the set $\bigcup_{H^{-\epsilon} \mathscr{X}} H$, where $H$ is the bounding hyperplane of $H^{-}$, is a closed set and consequently $\bigcap_{H^{-} \in \mathscr{C}}$ int $H^{-}$is a relatively open subset of $\bigcap_{H^{-} \in \mathscr{R}} H^{-}$.

THEOREM 4. A set $C$ in $E^{d}$ is the inner aperture of some convex subset of $E^{d}$ if and only if

$$
C=\boldsymbol{o} \cup \bigcap_{\mathscr{C}} \text { int. } H^{-}
$$

where $\mathscr{H}$ is an $F_{\sigma}$-collection of closed half-spaces and $\boldsymbol{o} \in H$, the bounding hyperplane of $\mathrm{H}^{-}$, for all $\mathrm{H}^{-} \in \mathscr{H}$.

REMARK. So, in particular, $C$ has to be a $G_{\tilde{r}}$-convex cone with apex the origin such that if $\boldsymbol{x} \in\{\mathrm{cl} . C\} \backslash C$ then the smallest exposed face $F(x)$ of cl. $C$ that contains $x$ is also contained in $\{$ cl. $C\} \backslash C$. In $E^{3}$ the converse is also true.

Proof. We shall assume that the theorem is true in $d-1$ dimensions, the theorem being trivial for $d=1$.

(i) Necessity. Let $C$ be the inner aperture of some convex set $D$ in $E^{d}$ where, since $\mathscr{J}(D)=\mathscr{I}(\mathrm{cl} . D)$ we may suppose that $D$ is 
closed. If $D=E^{d}$ then $C=E^{d}$ and, by convention,

$$
C=\bigcap_{\mathscr{C}} \text { int. } H^{-}=E^{d}
$$

where $\mathscr{H}$ is the empty set of closed half-spaces.

Otherwise $D \neq E^{d}$ and so possesses at least one hyperplane of support $M$ say with $D$ contained in the closed half-space $M^{-}$. We may suppose, without loss of generality, that $o \in M$. If $D$ contains a (maximal) linear subspace $L$ of dimension at least one then $L \subset M$ and

$$
D=F+L
$$

where $F$ is a closed convex subset of $L^{\perp}$. By the inductive assumption the inner aperture $\mathscr{J}(F)$ of $F$ can be written

$$
\mathscr{I}(F)=\boldsymbol{o} \cup \bigcap_{\mathscr{F} *} \text { int. } H^{*-}
$$

where $\mathscr{H}^{*}$ is a closed subset of the closed half-spaces in $L^{\perp}$. Then

$$
C=\boldsymbol{o} \cup \bigcap_{\mathscr{C}} \text { int. } H^{-}
$$

where $\mathscr{H}$ is the closed collection of closed half-spaces in $E^{d}$ formed by taking $H^{-}$in $\mathscr{H}$ if

$$
H^{-}=L+H^{*-}
$$

where $H^{*-} \in \mathscr{H}^{*}$.

If $D$ does not contain a line then the set of rays in $D$ is a closed convex cone $K$ which has a hyperplane of support say $\left\{x_{d}=0\right\}$ with

$$
K \cap\left\{x_{d}=0\right\}=\boldsymbol{o} .
$$

Let $\pi_{\nu}$ denote the hyperplane $x_{d}=\nu, \nu \geqq 0$. Let $l$ be a typical ray of $K$,

$$
\alpha_{\nu}(l)=\operatorname{dist} .\left\{\left(l \pi_{\nu}\right), \pi_{\nu}\left(E^{d} \backslash D\right)\right\},
$$

and

$$
\alpha(l)=\sup _{\nu \geqq 0} \alpha_{\nu}(l) .
$$

By considering two dimensional sections through $l$ it is easily verified that $\alpha_{\nu}(l)$ increases with $\nu$. Also

$$
l \subset C \text { if and only if } \alpha(l)=+\infty \text {. }
$$

So, if

$$
C_{i}=\{l: l \text { is a ray in } K, \alpha(l)>i\},
$$


then

$$
C=\bigcap_{i=1}^{\infty} C_{i}
$$

Now $C_{i} K, i=1,2, \cdots$ and

$$
K=o \cup \bigcap_{\mathscr{K}} \text { int. } H^{-}
$$

where $\mathscr{H}$ is the collection of closed half-spaces, whose bounding hyperplanes contain o, such that $K \backslash \boldsymbol{o} \subset$ int. $H^{-}$. If $\hat{K}=K \cap S^{d-1}$, let $\mathscr{H}_{j}^{*}$ denote the closed set of the closed half-spaces $H^{-}$,

$$
H^{-}=\{\boldsymbol{x}:\langle\boldsymbol{x}, \boldsymbol{u}\rangle \leqq 0\}
$$

where

$$
\langle-u, k\rangle \leqq-2^{-j}, \quad \text { for all } k \in \hat{K} \text {. }
$$

Then $\mathscr{H}=\bigcup_{j=1}^{\infty} \mathscr{H}_{j}^{*}$ and so, using (6), (7) it is enough to show that

$$
C_{i}=K \cap \bigcap_{\mathscr{K}_{i}} \text { int. } H^{-}
$$

where $\mathscr{H}_{i}$ is a closed collection of closed half-spaces of $E^{d}$ whose bounding hyperplanes goes through $o$.

Suppose now that $l$ is a ray of $K \backslash C_{i}$. Then

$$
\alpha(l) \leqq i
$$

For $j=1,2, \cdots$, there exist points $\boldsymbol{a}_{1}, \boldsymbol{a}_{2}, \cdots$, with $\boldsymbol{a}_{j} \in \pi_{j} \cap$ bdy. $D$ such that

$$
\left\|\boldsymbol{a}_{j}-\left\{\pi_{j} \cap l\right\}\right\| \leqq i
$$

Let $H_{j}$ denote a hyperplane of support to $D$ at $\boldsymbol{a}_{j}$, with $D \subset H_{j}^{-}$. As we may suppose that $K \neq \boldsymbol{o}, H_{j}$ is not parallel to the hyperplane $\pi_{1}$. So $H_{j} \cap \pi_{1}$ is a line in $\pi_{1}$. If we consider the two plane $\sigma_{j}$ through $l$ and $\boldsymbol{a}_{j}$ then $H_{j}$ meets $\sigma_{j}$ in a line $l_{j}$. As $l_{j}$ supports $\sigma_{j} \cap D$, it follows, using (8), that

$$
\left\|l_{j} \cap \pi_{1}-l \cap \pi_{1}\right\| \leqq i
$$

Consequently the $(d-2)$ affine space $\pi_{1} \cap H_{j}$ lies within a distance $i$ of $l \cap \pi_{1}$. So we may suppose, by picking subsequences if necessary, that $\pi_{1} \cap H_{j} \rightarrow \pi_{1} \cap H_{0}$ as $j \rightarrow \infty$ and $l_{j} \cap \pi_{1}$ tends to a point which, with a view to later developments, we denote by $l_{0} \cap \pi_{1}$. Let the line through the points $a_{j}$ and $l_{j} \cap \pi_{1}$ be $l_{j}^{*}, j=1,2, \ldots$. As (8), (9) hold, $l_{j}^{*}$ converges to a line $l_{0}$ through $l_{0} \cap \pi_{1}$ and parallel to $l$. Consequently $H_{j} \rightarrow H_{0}$ as $j \rightarrow \infty$. So $D \subset H_{0}^{-}$and 


$$
\left\|\pi_{\nu} \cap l_{0}-\pi_{\nu} \cap l\right\|=\beta \leqq i, \quad \text { if } \nu \geqq 0,
$$

$\beta$ a constant. We claim that

$$
H_{0}^{-}+\left\{\pi_{1} l-\pi_{1} l_{0}\right\}=H_{0}^{\prime-} \text { say },
$$

contains $K$ and $H_{0}^{\prime}$ supports $K$ and passes through $\boldsymbol{o}$. Certainly

$$
l \subset H_{0}^{\prime}
$$

and so $H_{0}^{\prime}$ passes through $o$. If there exists a ray $l^{*}$ in $K \backslash H_{0}^{\prime-}$, then $l^{*}$ meets $H_{0}$ which contradicts $D \subset H_{0}^{-}$.

Now let $\mathscr{H}_{i}$ denote those closed half-spaces $H^{-}$such that the bounding hyperplane $H$ supports $K$ and there exists a closed halfspace $H^{*-}$ containing $H^{-}$such that $H^{*}$ supports $D ; H^{*}$ is parallel to $H$ and a distance, in the hyperplane $\pi_{1}$, at most $i$ from $H$.

By (11),

$$
C_{i} \supset K \cap \bigcap_{\mathscr{C}_{i}} \text { int. } H^{-},
$$

where $\mathscr{H}_{i}$ is a closed set of closed half-spaces.

Conversely, if $l$ is a ray of

$$
K \backslash\left\{K \cap \bigcap_{\mathscr{C}_{i}} \operatorname{int} . H^{-}\right\}
$$

then there exists $H^{-}$in $\mathscr{H}_{i}$ such that $l \subset H$. Then there exists a closed half-space $H^{*-}$ which contains $D$ such that $H^{*}$ is parallel to $H$ and the distance between $H$ and $H^{*}$ is at most $i$. Consequently

$$
\alpha_{\nu}(l) \leqq i, \nu \geqq 0
$$

and so $l \not \subset C_{i}$. Hence

$$
C_{i} \subset K \cap \bigcap_{\mathscr{C}_{i}} \text { int. } H^{-}
$$

Combining (12) and (3),

$$
C_{i}=K \cap \bigcap_{\mathscr{C}_{i}} \text { int. } H^{-}
$$

which completes the proof of the necessity of the conditions.

(ii) Sufficiency. Suppose now that

$$
C=\boldsymbol{o} \cup \bigcap_{\mathscr{R}} \text { int. } H^{-}
$$

where $\mathscr{H}$ is an $F_{\sigma}$-collection of closed half-spaces and $\boldsymbol{o} \in H$ for all $H^{-} \in \mathscr{H}$. So we may write $\mathscr{H}=\bigcup_{i=1}^{\infty} \mathscr{H}_{i}$ where the $\mathscr{H}_{i}$ form an increasing sequence of closed collections.

Consider the closed convex cone 


$$
C_{0}=\operatorname{cl} . C=\bigcap_{\mathscr{C}} H^{-} .
$$

If $C_{0}=E^{d}$ then $C=E^{d}$ and $C$ is its own inner aperture. Otherwise $C_{0}$ possesses one hyperplane of support $M$ through $o$ with $C_{0}$ contained in the closed half-space $M^{-}$. If $M \cap C_{0}$ contains a maximal linear subspace $L$ of dimension at least 1 then we may write $C_{0}=F+L$ where $F$ is a proper closed convex cone in $L$. Notice that $L \subset H$ for each $H^{-} \in \mathscr{H}$ and consequently we may write

$$
H^{-}=L+H^{*-} \text { for each } H^{-} \in \mathscr{C},
$$

where $H^{*-}$ is a closed half-space in $L$ whose bounding hyperplane $H^{*}$ passes through o. Consequently

$$
C=\boldsymbol{o} \cup\left\{\left\{\boldsymbol{n} \text { int. } H^{*--}\right\}+L\right\} \text {. }
$$

By the inductive assumption, there exists a closed convex set $D^{*}$ in $L$ such that

$$
o \cup \bigcap_{\mathscr{H}} \text { int. } H^{*-}
$$

is the inner aperture of $D^{*}$ in $L$. Let

$$
D=D^{*}+L
$$

and then $C$ is the inner aperture of $D$.

Henceforth therefore we may suppose that $C_{0}$ is a proper closed convex cone in $E^{d}$ i.e., $C_{0}$ does not contain a line and we can also suppose that the ray

$$
X_{d}^{+}=\left\{\left(0, \cdots, 0, x_{d}\right), x_{d} \geqq 0\right\}
$$

is in $C_{0}$ and that the hyperplane $\pi_{0}=\left\{x_{d}=0\right\}$ supports $C_{0}$ with $\pi_{0} \cap C_{0}=$ o. Then, as for $K$ in the proof of necessity,

$$
C_{0}=\boldsymbol{o} \cup \bigcap_{\mathscr{C}_{0}} \text { int. } H^{-}
$$

where $\mathscr{C}_{0}$ is a closed set of closed half-spaces whose bounding hyperplanes pass through $o$. We may suppose that

$$
\mathscr{H}_{0} \subset \mathscr{H}_{1} \subset \mathscr{H}_{2} \subset \cdots
$$

and let

$$
C_{i}=\boldsymbol{o} \cup \bigcap_{\mathscr{C}_{i}} \text { int. } H^{-}, \quad i=0,1,2, \cdots .
$$

We shall produce inductively a nested sequence of closed convex sets $\left\{C_{i}^{*}\right\}_{i=0}^{*}$ such that $C_{i}$ is the inner aperture of $C_{i}^{*}$ and indeed 


$$
C_{i+1}^{*}=C_{i}^{*} \cap \bigcap_{\mathscr{C}_{i}} H^{*-}, i \geqq 0
$$

where, if $H^{-} \in \mathscr{H}_{i}$ then $H^{*-}$ is that closed half-space containing $H^{-}$ such that $H^{*}$ and $H$ are parallel and at a distance $i$ apart in the hyperplane $\pi_{1}$.

We begin the induction by taking

$$
C_{0}^{*}=\left\{\boldsymbol{x}=\left(x_{1}, \cdots, x_{d}\right), x_{d} \geqq 0 \text { and dist. }\left(\boldsymbol{x}, C_{0} \cap \pi_{x_{d}}\right) \leqq x_{d}^{1 / 2}\right\} .
$$

Clearly $C_{0}^{*}$ is closed and it is convex since, from above, $C_{0}^{*} \cap \pi_{\nu}$ is convex, $\nu \geqq 0$ and so $C_{0}^{*}$ cannot possess a point of concavity. We shall show that

$$
\mathscr{I}\left(C_{0}^{*}\right)=C_{0}
$$

First notice that if $\boldsymbol{u}=\left(u_{1}, \cdots, u_{d}\right)$ is a unit vector in $C_{0}$ then $u_{d}>$ 0 . So, if $l=\{\lambda u: \lambda \geqq 0\}$ is the corresponding ray in $C_{0}$

$$
\theta_{\lambda}=\alpha_{\lambda u_{d}}(l) \geqq \sqrt{\lambda u_{d}}>0 \text {. }
$$

So, if $m$ is a positive number

$$
\theta_{\lambda} \geqq m
$$

provided $m^{2} / u_{d} \leqq \lambda$. It is an almost immediate consequence of (16) that $l \subset \mathscr{J}\left(C_{0}^{*}\right)$ and hence $C_{0} \subset \mathscr{J}\left(C_{0}^{*}\right)$.

Suppose next that the ray

$$
l^{\prime}=\{\lambda v, \lambda \geqq 0\}
$$

is not in $C_{0}$. If $v_{d} \leqq 0$ then $\lambda \boldsymbol{v} \notin C_{0}^{*}$ for all $\lambda>0$ and then certainly $l^{\prime} \not \subset \mathscr{F}\left(C_{0}^{*}\right)$. If $v_{d}>0$ then $l^{\prime} \cap \pi_{\nu}$ is a single point for each $\nu \geqq 0$ and there exists $\eta>0$ such that

$$
\text { dist. }\left(v, C_{0} \cap \pi_{v_{d}}\right)>\eta \text {. }
$$

So

$$
\text { dist. }\left(\lambda v, C_{0} \pi_{\lambda v_{d}}\right)>\lambda \eta \text {. }
$$

But, if $l^{\prime} \subset \mathscr{S}\left(C_{0}^{*}\right)$ then, in particular, $\lambda \boldsymbol{v} \in C_{0}^{*}$ for each $\lambda \geqq 0$. So

$$
\text { dist. }\left(\lambda \boldsymbol{v}, C_{0} \pi_{\lambda v_{d}}\right) \leqq\left(\lambda v_{d}\right)^{1 / 2}, \lambda \geqq 0 .
$$

However, provided $\lambda>v_{d} / \eta^{2}$ it follows from (17) that (18) is false. Consequently $l^{\prime} \not \subset \mathscr{I}\left(C_{0}^{*}\right)$ which establishes (15).

Suppose inductively that for some $m \geqq 1$ we have constructed $m$ closed convex sets $C_{0}^{*}, \cdots, C_{m-1}^{*}$ in $E^{d}$ with $C_{i}$ being the inner aperture of $C_{i}^{*}, i=0, \cdots, m-1$. Indeed, 
ON THE INNER APERTURE AND INTERSECTIONS OF CONVEX SETS 231

$$
C_{i+1}^{*}=C_{i}^{*} \cap \bigcap_{\mathscr{C}} H_{i+1}^{*-}, \quad i=0,1, \cdots, m-2,
$$

where, if $H^{-} \in \mathscr{H}_{i+1}$ then $H^{*-}$ is that closed half-space containing $H^{-}$ such that $H^{*}$ and $H$ are parallel and at a distance $i+1$ apart in the plane $\pi_{1}$.

For each $H^{-} \in \mathscr{H}_{m}$, let $H^{*-}$ be that closed half-space containing $H^{-}$such that $H^{*}$ and $H$ are parallel and at a distance $m$ apart in the plane $\pi_{1}$. Define

$$
C_{m}^{*}=C_{m-1}^{*} \cap \bigcap_{m} H^{*-}
$$

We claim that the inner aperture of $C_{m}^{*}$ is $C_{m}$ i.e.,

$$
\mathscr{I}\left(C_{m}^{*}\right)=C_{m} \text {. }
$$

If $l$ is a ray of $C_{0}$ not in $C_{m}$ then $l$ is in some hyperplane $H$ where $H^{-} \in \mathscr{H}_{m}$. Consequently, by considering the corresponding closed halfspace $H^{*-}$, we deduce that $\alpha(l) \leqq m$, and so $l \not \subset \mathscr{F}\left(C_{m}^{*}\right)$. Hence $\mathscr{J}\left(C_{m}^{*}\right) \subset C_{m}$.

On the other hand, suppose that $l \in C_{m}$. That the set

$$
\bigcup_{\mathscr{K}} H^{*}=H_{m} \text { say }
$$

is a closed set and does not meet the ray $l \backslash \boldsymbol{o}$. As each hyperplane $H$, with $H^{-} \in \mathscr{H}_{m}$, passes through $\boldsymbol{o}$, it follows that

$$
\text { dist. }\left(l \cap \pi_{\nu}, H_{m}\right) \longrightarrow+\infty \text { as } \nu \longrightarrow+\infty \text {. }
$$

Also $l \in \mathscr{J}\left(C_{m-1}^{*}\right)$ and so

$$
\text { dist. }\left(l \cap \pi_{\nu}, E^{d} \backslash C_{m-1}^{*}\right) \longrightarrow+\infty \text { as } \nu \longrightarrow+\infty \text {. }
$$

Consequently using (20), (22), (23),

$$
\text { dist. }\left(l \cap \pi_{\nu}, E^{d} \backslash C_{m}^{*}\right) \longrightarrow+\infty \text { as } \nu \longrightarrow+\infty \text {. }
$$

Therefore, $l \subset \mathscr{J}\left(C_{m}^{*}\right)$ and so $C_{m} \subset \mathscr{I}\left(C_{m}^{*}\right)$ which completes the verification of (21).

The results (20), (21) verify (19) for $m$ and we can now suppose that the $C_{m}^{*}$ have been defined so that (20), (21) hold for $m=0,1,2$, ... Define

$$
C^{*}=\bigcap_{m=0}^{\infty} C_{m}^{*}
$$

and we shall show that $\mathscr{F}\left(C^{*}\right)=C$.

Suppose that $l$ is a ray of $C_{0}$ not in $\mathscr{F}\left(C^{*}\right)$. Then there exists $m$ such that $\alpha_{\nu}(l) \leqq m, \nu \geqq 0$. So $l$ is not in $\mathscr{S}\left(C_{m+1}^{*}\right)=C_{m+1}$. Consequently $l$ is not in $C$. So $C \subset \mathscr{I}\left(C^{*}\right)$. 
On the other hand, suppose that $l$ is a ray of $C_{0}$ which is not in $C$. Then $l$ is not in $C_{m}$ for some $m \geqq 0$. So

$$
l \not \subset \mathscr{I}\left(C_{m}^{*}\right) \supset \mathscr{I}\left(C^{*}\right) \text {. }
$$

Hence $\mathscr{I}\left(C^{*}\right) \subset C$ and this finally establishes that

$$
\mathscr{J}\left(C^{*}\right)=C
$$

which completes the proof of Theorem 4 .

\section{REFERENCES}

1. J. E. Lewis, On a problem of uniqueness arising in connection with a neurophysical control mechanism, Submitted to Information and Control.

2. Z. A. Melzak, On a uniqueness theorem and its application to a neurophysical control mechanism, Information and Control, 5 (1962), 163-172.

3. F. Ratcliff and H. K. Hartline, The response of Limus optic nerve fibers to patterns of illumination on the receptor mosaic, J. Genl. Physiol., 42 (1959), 1241-1255.

4. L. A. Santaló, Un teorema sobre conjuntos de paralelepipedos de aristas paralelas, Publ. Inst. Mat. Univ. Nac. Litoral, 2 (1940), 49-60 and 3 (1942), 202-210.

Received April 30, 1973 and in revised form August 21, 1973. Research supported by the National Research Council of Canada, while visiting the University of British Columbia.

University College LONDON 


\section{PACIFIC JOURNAL OF MATHEMATICS}

EDITORS

RICHARD ARENS (Managing Editor)

University of California

Los Angeles, Calıfornia 90024

R. A. Beaumont

University of Washington

Seattle, Washington 98105
J. DugundJI

Department of Mathematics

University of Southern California

Los Angeles, California 90007

D. Gilbarg and J. Milgram

Stanford University

Stanford, California 94305

\section{ASSOCIATE EDITORS}
E. F. BECKENBACH
B. H. NeumanN
F. WOLF
K. YOSHIDA

\section{SUPPORTING INSTITUTIONS}

UNIVERSITY OF BRITISH COLUMBIA

CALIFORNIA INSTITUTE OF TECHNOLOGY

UNIVERSITY OF CALIFORNIA

MONTANA STATE UNIVERSITY

UNIVERSITY OF NEVADA

NEW MEXICO STATE UNIVERSITY

OREGON STATE UNIVERSITY

UNIVERSITY OF OREGON

OSAKA UNIVERSITY
UNIVERSITY OF SOUTHERN CALIFORNIA

STANFORD UNIVERSITY

UNIVERSITY OF TOKYO

UNIVERSITY OF UTAH

WASHINGTON STATE UNIVERSITY

UNIVERSITY OF WASHINGTON

AMERICAN MATHEMATICAL SOCIETY NAVAL WEAPONS CENTER 


\section{Pacific Journal of Mathematics}

\section{Vol. 55, No. $1 \quad$ September, 1974}

Robert Lee Anderson, Continuous spectra of a singular symmetric

differential operator on a Hilbert space of vector-valued functions . . . $\quad 1$

Michael James Cambern, The isometries of $L^{p}(X, K) \ldots \ldots \ldots \ldots \ldots . . \ldots$

R. H. Cameron and David Arne Storvick, Two related integrals over spaces of continuous functions ................................

Gary Theodore Chartrand and Albert David Polimeni, Ramsey theory and

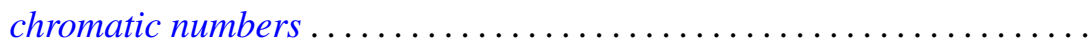

John Deryck De Pree and Harry Scott Klein, Characterization of collectively compact sets of linear operators ...................

John Deryck De Pree and Harry Scott Klein, Semi-groups and collectively compact sets of linear operators ....................... 55

George Epstein and Alfred Horn, Chain based lattices.............. 65

Paul Erdős and Ernst Gabor Straus, On the irrationality of certain series . . 85

Zdeněk Frolík, Measurable uniform spaces................... 93

Stephen Michael Gagola, Jr., Characters fully ramified over a normal

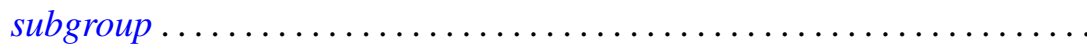

Frank Larkin Gilfeather, Operator valued roots of abelian analytic

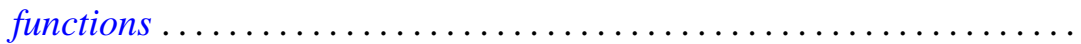

D. S. Goel, A. S. B. Holland, Cyril Nasim and B. N. Sahney, Best approximation by a saturation class of polynomial operators

James Secord Howland, Puiseux series for resonances at an embedded

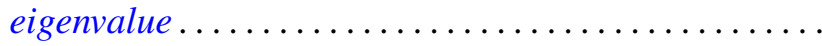

David Jacobson, Linear GCD equations .................

P. H. Karvellas, A note on compact semirings which are multiplicative

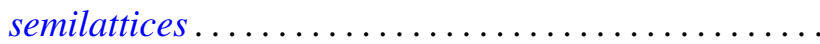

Allan Morton Krall, Stieltjes differential-boundary operators. II . .

D. G. Larman, On the inner aperture and intersections of convex sets

S. N. Mukhopadhyay, On the regularity of the $P^{n}$-integral and its application to summable trigonometric series ....... .

Dwight Webster Read, On $(J, M, m)$-extensions of Boolean algebras ....

David Francis Rearick, Multiplicativity-preserving arithmetic power series.

Indranand Sinha, Characteristic ideals in group algebras

Charles Thomas Tucker, II, Homomorphisms of Riesz spaces . . .

Kunio Yamagata, The exchange property and direct sums of indecomposable injective modules. 